\title{
Past and Present ARDS Mortality Rates: A Systematic Review
}

\author{
Jan Máca MD, Ondřej Jor MD, Michal Holub MD PhD, Peter Sklienka MD, \\ Filip Burša MD PhD, Michal Burda PhD, Vladimír Janout MD CSc, \\ and Pavel Ševčík MD CSc
}

\author{
Introduction \\ Methods \\ Results \\ Study Selection and Characteristics \\ Mortality Rates in Relation to Study Methodology and Time Period \\ Trends and Recent Mortality Rates \\ Discussion \\ Conclusions
}

\begin{abstract}
ARDS is severe form of respiratory failure with significant impact on the morbidity and mortality of critical care patients. Epidemiological data are crucial for evaluating the efficacy of therapeutic interventions, designing studies, and optimizing resource distribution. The goal of this review is to present general aspects of mortality data published over the past decades. A systematic search of the MEDLINE/PubMed was performed. The articles were divided according to their methodology, type of reported mortality, and time. The main outcome was mortality. Extracted data included study duration, number of patients, and number of centers. The mortality trends and current mortality were calculated for subgroups consisting of inhospital, ICU, 28/30-d, and 60-d mortality over 3 time periods (A, before 1995; B, 1995-2000; $\mathrm{C}$, after 2000). The retrospectivity and prospectivity were also taken into account. Moreover, we present the most recent mortality rates since 2010 . One hundred seventy-seven articles were included in the final analysis. General mortality rates ranged from 11 to $87 \%$ in studies including subjects with ARDS of all etiologies (mixed group). Linear regression revealed that the study design (28/30-d or 60-d) significantly influenced the mortality rate. Reported mortality rates were higher in prospective studies, such as randomized controlled trials and prospective observational studies compared with retrospective observational studies. Mortality rates exhibited a linear decrease in relation to time period $(P<.001)$. The number of centers showed a significant negative correlation with mortality rates. The prospective observational studies did not have consistently higher mortality rates compared with randomized controlled trials. The mortality trends over 3 time periods (before 1995, 1995-2000, and after 2000) yielded variable results in general ARDS populations. However, a mortality decrease was present mostly in prospective studies. Since 2010, the overall rates of in-hospital, ICU, and 28/30-d and 60-d mortality were 45, 38, 30, and 32\%, respectively. Key words: acute lung injury; ARDS; mortality; outcome; clinical trial. [Respir Care 2017;62(1):113-122. (C) 2017 Daedalus Enterprises]
\end{abstract}




\section{Mortality of ARDS: A Systematic Review}

\section{Introduction}

ARDS is a highly heterogeneous life-threatening severe form of acute respiratory failure. It is characterized by an acute onset of hypoxemia refractory to oxygen treatment and the presence of bilateral pulmonary infiltrates on chest radiograph. ${ }^{1-4}$ The syndrome is based on alveolar-capillary membrane injury followed by the development of pulmonary edema, which cannot be fully explained by heart failure. ${ }^{1-3}$ ARDS is usually accompanied by multipleorgan system failure and therefore substantially contributes to high morbidity and mortality in critical care. ${ }^{5,6}$ It increases the cost of treatment of critical care patients by prolonging ICU and in-hospital stay. Reliable epidemiologic data concerning ARDS are important for all critical care physicians. These data facilitate clinical decision making, with implications for the efficacy of current therapeutic interventions, and suggest ways for their future development. ${ }^{7}$ Moreover, the data help us to better assess the present clinical and economic impact of the condition and may lead to more appropriate resource distribution.

The history of ARDS should be divided into 3 main periods: (1) before 1995 (before publication of the American-European Consensus Conference [AECC] criteria), (2) from 1995 to 2000 (implementation of the AECC criteria and development of lung-protective mechanical ventilation), and (3) from 2001 to the present (the era of lung-protective mechanical ventilation).

The main goals of this systematic review of the literature are to highlight the complexity of ARDS mortality reporting, to outline the trends over the last 2 decades, and

Drs Máca, Jor, Sklienka, Burša, and Ševčík are affiliated with the Department of Anesthesiology and Intensive Care Medicine, University Hospital of Ostrava, Ostrava, Czech Republic. Drs Máca, Sklienka, Burša, and Ševčík are affiliated with the Department of Intensive Care Medicine and Forensic Studies, Faculty of Medicine, University of Ostrava, Ostrava, Czech Republic. Dr Holub is affiliated with the Department of Infectious Diseases, First Faculty of Medicine, Charles University in Prague and Military University Hospital, Prague, Czech Republic. Dr Burda is affiliated with the Institute for Research and Applications of Fuzzy Modeling, Centre of Excellence IT4Innovations, University of Ostrava, Ostrava, Czech Republic. Dr Janout is affiliated with the Department of Epidemiology and Public Health, Faculty of Medicine, University of Ostrava, Ostrava, Czech Republic.

This study was supported by Grants SVV 260026 and MO 1012, Charles University Prague. The authors have disclosed no conflicts of interest.

Supplementary material related to this paper is available at http:// www.rcjournal.com.

Correspondence: Jan Máca MD, Department of Anesthesiology and Intensive Care Medicine, University Hospital, Ostrava, 17. listopadu 1790, 70852 Ostrava-Poruba, Czech Republic. E-mail: jan.maca@fno.cz.

DOI: $10.4187 /$ respcare. 04716 to estimate current mortality rates of ARDS, suggesting benchmarks in various clinical trial designs. Moreover, uncertainties and controversies regarding the topic are assessed, and possible explanations are presented.

\section{Methods}

We used the PRISMA (Preferred Reporting Items for Systematic Reviews and Meta-Analyses) statement as a guide. ${ }^{8}$ A computerized search through the online web database MEDLINE/PubMed was performed. The search was performed by the MeSH (Medical Subject Headings) system using the key words "acute lung injury" AND "acute respiratory distress syndrome" AND "mortality" AND "outcome" AND "incidence" AND "clinical trial." The bibliographies of the retrieved articles were also reviewed. Human studies with $>20$ subjects published from January 1994 to January 2015 were taken into consideration. NonEnglish articles were included, provided that they contained all required data. Two independent reviewers (MJ and JO) screened the titles, abstracts, and methodological quality of the articles with a high degree of agreement (93.2\%). All eligible articles were retrieved in full text (see online supplement I at http://www.rcjournal.com).

The data collected in a data sheet were as follows: study group characteristics (specific vs mixed ARDS population). We defined a mixed population as a group of subjects with ARDS irrespective of etiology. A group of subjects enrolled into the study primarily due to the presence of a unique diagnosis (ie, multiple trauma, sepsis, burns, military injury) or intervention (ie, extracorporeal oxygenation, high-frequency ventilation) was defined as the specific ARDS population. The specific population was omitted from final analysis to improve generalization of the results. Additional extracted data included number of centers, number of enrolled patients with ARDS, time of enrollment (in months), time period when the study was started and performed, type of mortality, and absolute value of the reported mortality rate. The characteristics of the specific ARDS populations are described in Table 1.

Studies with mixed ARDS populations were divided into 3 groups according to the study's methodology: (1) prospective observational, (2) retrospective observational, and (3) randomized controlled trials (RCTs). In prospective and retrospective observational studies, we considered all enrolled subjects with ARDS; in RCTs, we screened only the mortality rates in the control (non-interventional) group to obtain a set of subjects comparable with the observational ones and to avoid possible influence of the intervention.

The reported mortality rates were also categorized by type: in-hospital, ICU, 28-d, 30-d, 60-d, 90-d, 365-d, etc. Some papers reported more than one type of mortality rate. For further analysis, only the most frequent mortality types 
Table 1. Characteristics of the Specific ARDS Populations

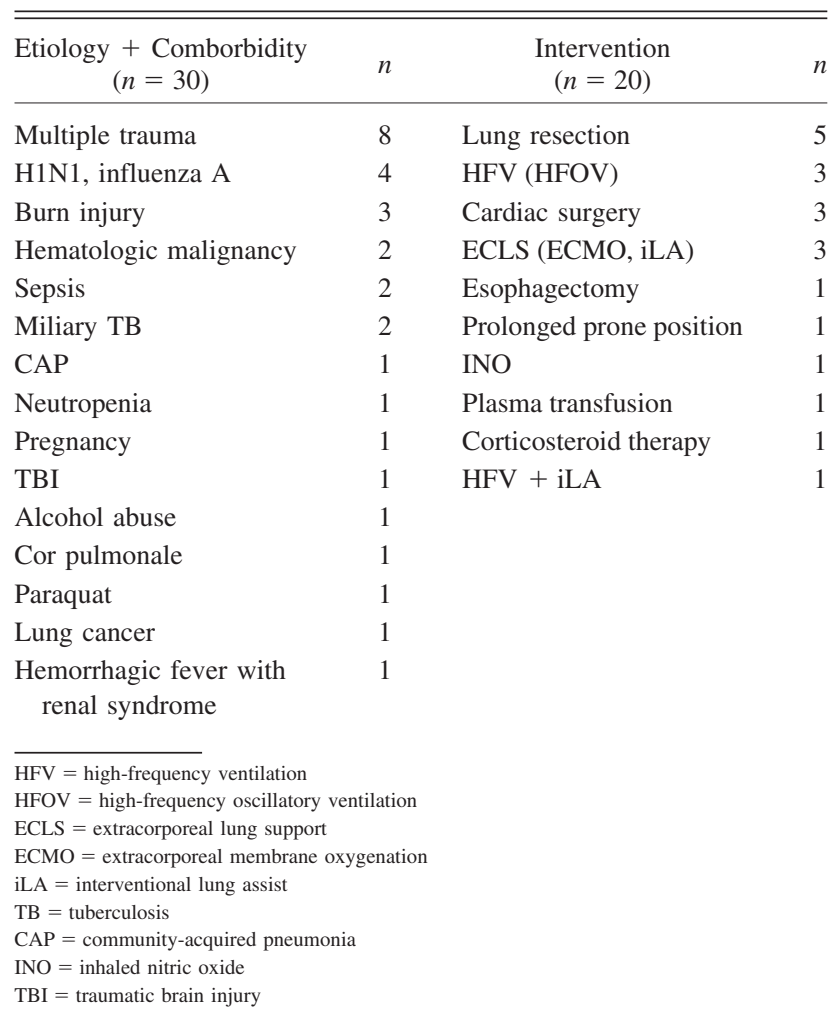

were selected, and they were analyzed separately: in-hospital, ICU, 28/30-d mortality (note: 28-d mortality and 30-d mortality were considered together), and 60-d mortality. The rest of the mortality types that were reported rarely in the papers were omitted from further analysis.

To assess the aspect of time, we subdivided the main groups (prospective observational studies, retrospective observational studies, and RCTs) into 3 subgroups in relation to the time of the initiation and performance of the study: (1) before 1995, before the publication of the AECC criteria (subgroup A), (2) from 1995 to 2000, when the highlighted ARDS network study ${ }^{9}$ was published (subgroup B), and (3) from 2001 to the present (subgroup C). Overall mortality rates (weighted by number of subjects) and their trends over time were calculated for all subgroups. Moreover, we separately calculated the most recent cumulative mortality rates since 2010 .

To resolve the bias stemming from different sample sizes among the studies, aggregated mortality rates were computed as a weighted average of the mortality rate reported in each study, with the weight being the number of subjects involved. To assess simultaneous influences of the independent variables on weighted mortality, a binomial family generalized linear model was fitted to the data. Ordered categories A, B, and C, which represent the time periods, were encoded using orthogonal polynomial cod- ing, which allows trends to be captured in a linear or quadratic manner.

The terminology and classification of acute respiratory failure used in this review and during the search of databases respected the usual nomenclature in the respective period of time (eg, the AECC criteria). ${ }^{1,2}$ The authors are aware of the current recommendations to use only the term ARDS according to the recently published Berlin definition. ${ }^{3}$

The certified statistician (MB) used R 3.2.0 (R Foundation for Statistical Computing, Vienna, Austria) for statistical analysis and for creating the figures and tables presented in this study. ${ }^{10}$ The data set obtained underwent statistical assessment for quality. Missing values, outliers, and other suspicious values were noted and double-checked. The analysis of the data set dealt mainly with mortality rates (ie percentage rates of occurrence of some event, which is a random variable with binomial distribution). Therefore, to resolve the bias stemming from different sample sizes among the studies, aggregated mortality rates were obtained by computing arithmetic means of reported mortality rates weighted by numbers of subjects included in the studies. To assess simultaneous influences of multiple variables on weighted mortality, a binomial family generalized linear model was fitted to the data.

\section{Results}

\section{Study Selection and Characteristics}

The initial search identified 1,265 potentially eligible articles. A total of 1,096 papers were excluded for the following reasons: 843 were considered to be not relevant mainly because of their topic, focus, and methodological quality; 85 involved only pediatric subjects; 20 were preventive; 25 used an inappropriate definition; 6 were missing required data; 94 were reviews/meta-analyses/post hoc analyses; 5 were congress proceedings/reports; 16 were case reports or included fewer than the required number of subjects; and 2 were currently recruiting for trials. The bibliographies of the retrieved articles contained 8 articles that met the inclusion criteria and were additionally considered for this systematic review. In total, the resulting 177 papers reported 189 enrollment intervals (historical cohorts), which we treated as separate studies (see Fig. 1).

\section{Mortality Rates in Relation to Study Methodology and Time Period}

In general, mortality rates ranged from 13 to $80 \%$ in the specific group and from 11 to $87 \%$ in the mixed group. More studies screened mixed ARDS populations than specific ARDS populations ( $n=139$ vs $n=50$, respectively). Many articles report multiple mortality rates. The list of 


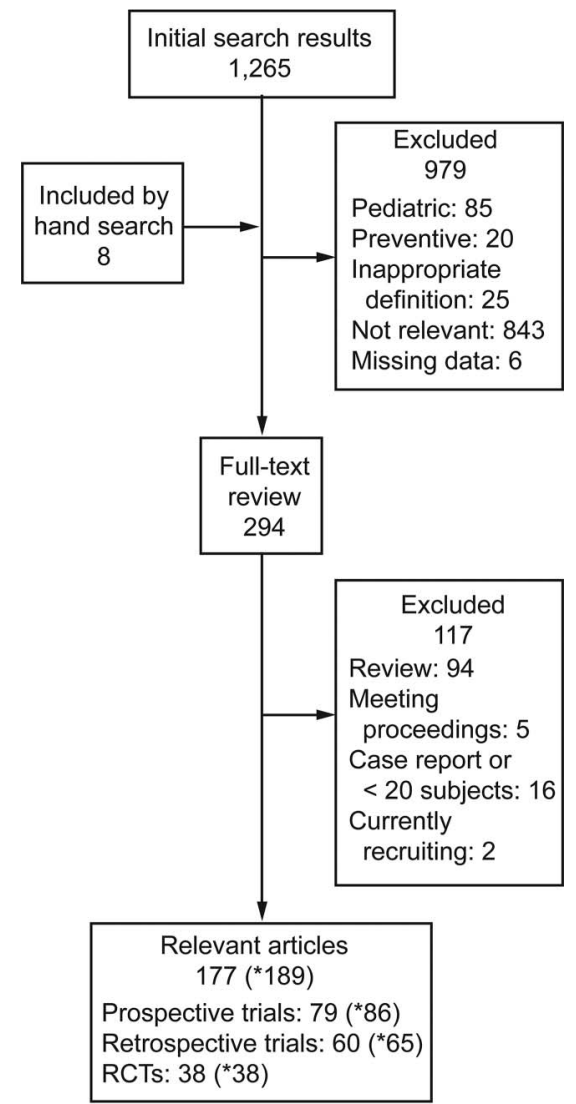

Fig. 1. Flow chart. RCT = randomized controlled trial. Number of studies (*) may be greater than the number of articles because some articles reported results of studies that can be considered separately (eg, 2 or 3 independent historical cohorts).

specific ARDS populations is displayed in Table 1. The total number of subjects in all selected studies was 38,351 .

Among the mixed ARDS population, the most frequently reported type of mortality rate was in-hospital (no. $=50$ ), followed by ICU mortality (no. = 34), 28/30-d mortality $($ no. $=31)$, and $60-\mathrm{d}$ mortality $($ no. $=16)$. Only studies with mixed population were selected for further analysis (no. $=106$ ). The total number of subjects in the mixed group was 25,966. Figure 2 depicts the number of studies and the total number of subjects involved in studies categorized by mortality type and study methodology. Table 2 shows in detail the number of studies, number of subjects, and average mortality rates related to study methodology, mortality type, and time period.

The generalized linear model with the binomial family was used to assess simultaneous influences of various variables (number of centers, number of months, and study design) on mortality rates. A logarithm of odds ratios (logits) was predicted using the linear regression (see online supplement II). The mortality is significantly influenced by the study design. If the type of mortality is $28 / 30-\mathrm{d}$ or $60-\mathrm{d}$, the logits (as surrogate for the mortality rate) de- crease significantly by 0.44 or 0.45 (both $P<.001$, respectively). If the study is prospective (ie, prospective observational studies or RCTs), then the reported mortality increases by 0.2 logits $(P<.001)$.

The orthogonal polynomial contrasts allowed us to evaluate the time period linearity and quadraticity, assessing the influence of time period on the reported mortality rates. The mortality rate has a linear decreasing tendency by logit 0.15 in relation to time period $(P<.001)$. Quadratic influences of time period were not identified as statistically significant.

The mortality rates showed significant dependence on the number of centers. With an increasing number of centers included in the studies, the reported mortality rate decreases by 0.003 logits $(P<.001)$. A dependence of mortality rates on the length of the studies (months) was not found $(P=.57)$.

Table 3 shows the detailed mortality rates in defined time periods (A < 1995; B 1995-2000; C > 2000) grouped by prospectivity or retrospectivity. Lower mortality was reported in retrospective mode in almost all calculable subgroups (9 of 10). A significant difference was found for 28/30-d mortality rates in period A $(P<.001)$, in-hospital mortality in periods $\mathrm{B}$ and $\mathrm{C}(P<.001$, and $P<.001$, respectively), and ICU mortality rates in period $\mathrm{B}(P=$ .018) (4 of 10).

The differences between prospective modes of study subgroups (prospective observational studies and RCTs) are described in Table 4. We found higher mortality in prospective observational studies compared with RCTs in 7 of 9 calculable subgroups with significance for in-hospital morality in periods B and C (both $P<.001$, respectively), in 28/30-d mortality in the same periods $(P<.001$ and $P=.046$, respectively), and in 60-d mortality in pe$\operatorname{riod} \mathrm{C}(P<.001)(5$ of 9$)$.

\section{Trends and Recent Mortality Rates}

A detailed analysis of trends (see Fig. 3) shows that the mortality rate decline is statistically significant for in-hospital mortality in retrospective observational studies, ICU mortality in RCTs, $28 / 30$-d mortality in prospective observational studies and RCTs, and 60-d mortality in RCTs. There was a mild to moderate decrease tendency for ICU mortality in prospective observational studies and for 60-d mortality in RCTs. There was no significant change in mortality for in-hospital RCTs, ICU mortality in retrospective observational studies, and 28/30-d mortality in retrospective observational studies. The significant increase in mortality was observed for in-hospital mortality in prospective observational studies. The data for calculation of the 60-d mortality trend in retrospective observational studies was missing due to a lack of studies reporting the particular type of mor- 

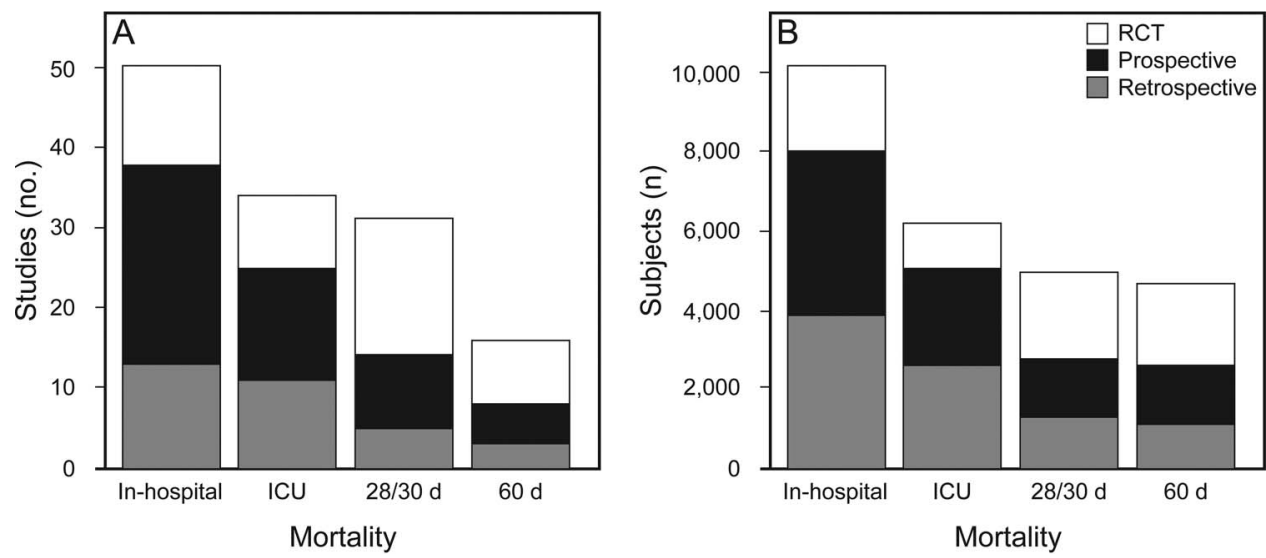

Fig. 2. Number of studies and participating subjects, grouped by study type and reported type of mortality. RCT = randomized controlled trial.

Table 2. Number of Studies Related to Study Methodology, Mortality Type, and Time Period

\begin{tabular}{|c|c|c|c|c|c|c|c|c|c|c|c|c|c|}
\hline \multirow{2}{*}{$\begin{array}{l}\text { Mortality } \\
\text { Type }\end{array}$} & \multirow[b]{2}{*}{ Period } & \multicolumn{3}{|c|}{ Retrospective } & \multicolumn{3}{|c|}{ Prospective } & \multicolumn{3}{|c|}{ RCTs } & \multicolumn{3}{|c|}{ All } \\
\hline & & $\begin{array}{c}\text { Studies } \\
\text { (no.) }\end{array}$ & $\begin{array}{l}\text { Subjects } \\
(N)\end{array}$ & $\%$ & $\begin{array}{c}\text { Studies } \\
\text { (no.) }\end{array}$ & $\begin{array}{c}\text { Subjects } \\
(N)\end{array}$ & $\%$ & $\begin{array}{c}\text { Studies } \\
\text { (no.) }\end{array}$ & $\begin{array}{l}\text { Subjects } \\
\qquad(N)\end{array}$ & $\%$ & $\begin{array}{c}\text { Studies } \\
\text { (no.) }\end{array}$ & $\begin{array}{l}\text { Subjects } \\
(N)\end{array}$ & $\%$ \\
\hline \multirow[t]{4}{*}{ In-hospital } & A & 6 & 900 & 50 & 6 & 440 & 49 & 1 & 13 & 43 & 13 & 1,353 & 50 \\
\hline & B & 6 & 2,719 & 38 & 11 & 2,420 & 46 & 5 & 925 & 38 & 22 & 6,064 & 41 \\
\hline & $\mathrm{C}$ & 1 & 200 & 32 & 8 & 1,368 & 51 & 6 & 1,128 & 41 & 15 & 2,696 & 46 \\
\hline & All & 13 & 3,819 & 40 & 25 & 4,228 & 48 & 12 & 2,066 & 40 & 50 & 10,113 & 43 \\
\hline \multirow[t]{4}{*}{ ICU } & $\mathrm{A}$ & 3 & 436 & 35 & 1 & 27 & 52 & 0 & 0 & N/A & 4 & 463 & 36 \\
\hline & $\mathrm{B}$ & 5 & 1,914 & 39 & 9 & 1,523 & 42 & 4 & 153 & 50 & 18 & 3,590 & 41 \\
\hline & $\mathrm{C}$ & 3 & 260 & 36 & 4 & 929 & 40 & 5 & 966 & 40 & 12 & 2,155 & 40 \\
\hline & All & 11 & 2,610 & 38 & 14 & 2,479 & 42 & 9 & 1,119 & 41 & 34 & 6,208 & 40 \\
\hline \multirow[t]{4}{*}{ 28/30-d } & $\mathrm{A}$ & 1 & 345 & 27 & 0 & 0 & N/A & 4 & 449 & 41 & 5 & 794 & 35 \\
\hline & B & 2 & 191 & 31 & 5 & 789 & 42 & 5 & 466 & 31 & 12 & 1,446 & 37 \\
\hline & $\mathrm{C}$ & 2 & 809 & 28 & 4 & 606 & 34 & 8 & 1,288 & 29 & 14 & 2,703 & 30 \\
\hline & All & 5 & 1,345 & 28 & 9 & 1,395 & 38 & 17 & 2,203 & 32 & 31 & 4,943 & 33 \\
\hline \multirow[t]{4}{*}{$60-d$} & A & 0 & 0 & N/A & 0 & 0 & N/A & 0 & 0 & N/A & 0 & 0 & N/A \\
\hline & B & 3 & 1,124 & 36 & 1 & 177 & 40 & 3 & 192 & 40 & 7 & 1,493 & 37 \\
\hline & $\mathrm{C}$ & 0 & 0 & N/A & 4 & 1,294 & 38 & 5 & 1,915 & 26 & 9 & 3,209 & 30 \\
\hline & All & 3 & 1,124 & 36 & 5 & 1,471 & 38 & 8 & 2,107 & 27 & 16 & 4,702 & 32 \\
\hline Total & & $25^{*}$ & 8,898 & 37 & $46^{*}$ & 9,573 & 43 & $35^{*}$ & 7,495 & 34 & $106^{*}$ & 25,966 & 39 \\
\hline \multicolumn{14}{|c|}{ 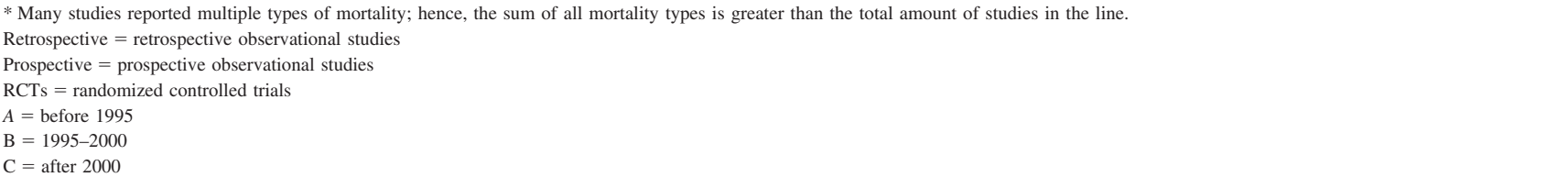 } \\
\hline
\end{tabular}

tality. Overall, 7 of the 12 subgroups showed a decrease $(58 \%)$ and 3 showed stagnation $(25 \%)$ of the mortality rate trend. Moreover, the mortality rate decrease was present in 6 prospective and one retrospective subgroup.

Table 5 summarizes the absolute values of the most recent mortality rates since 2010. Currently, the overall rates of reported in-hospital, ICU, 28/30-d, and 60-d mortality are $45,38,30$, and $32 \%$, respectively.

\section{Discussion}

Reporting of ARDS epidemiology is extremely heterogeneous. Its variability is based not only on differences between causal factors (type and intensity) and route of lung injury (pulmonary vs extrapulmonary ARDS) but also on genetic background, the presence of various complications (ie, secondary infections), and 
Table 3. Comparison of the Mortality Rates in Studies With Retrospective and Prospective Design in Different Time Periods

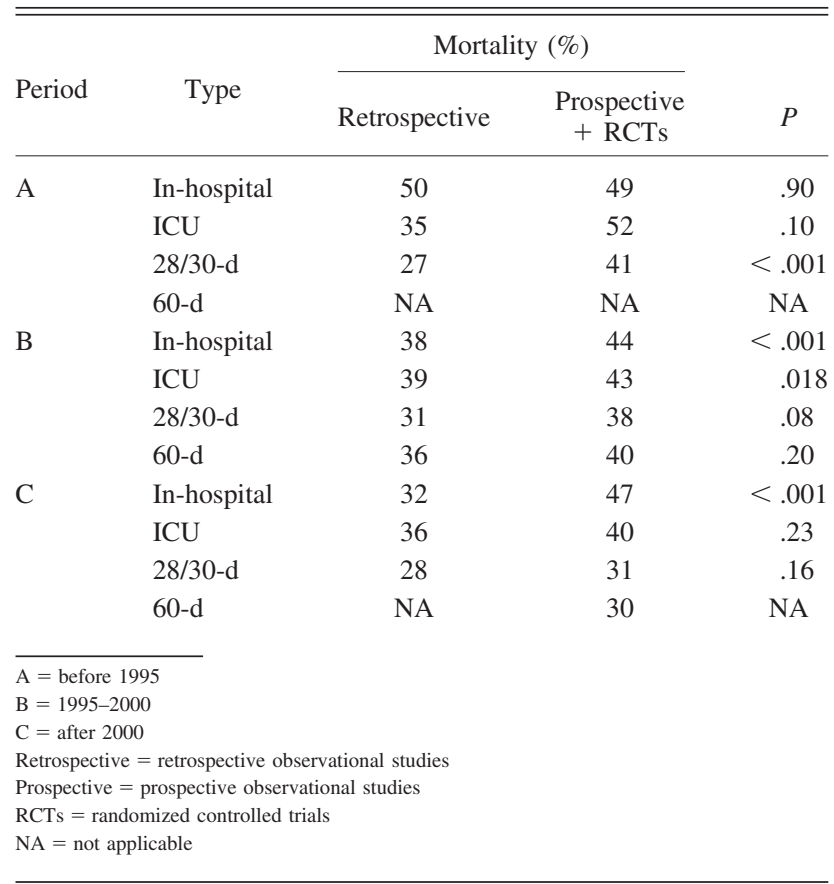

Table 4. Comparison of the Mortality Rates in Studies With Prospective Design in Different Time Periods

\begin{tabular}{|c|c|c|c|c|}
\hline \multirow{2}{*}{ Period } & \multirow{2}{*}{ Type } & \multicolumn{2}{|c|}{ Mortality (\%) } & \multirow{2}{*}{$P$} \\
\hline & & Prospective & RCTs & \\
\hline \multirow[t]{4}{*}{ A } & In-hospital & 49 & 43 & .86 \\
\hline & ICU & 52 & NA & NA \\
\hline & 28/30-d & NA & 41 & NA \\
\hline & $60-d$ & NA & NA & NA \\
\hline \multirow[t]{4}{*}{ B } & In-hospital & 46 & 38 & $<.001$ \\
\hline & ICU & 42 & 50 & .10 \\
\hline & $28 / 30$ & 42 & 31 & $<.001$ \\
\hline & 60 & 40 & 40 & $>.99$ \\
\hline \multirow[t]{4}{*}{$\mathrm{C}$} & In-hospital & 51 & 41 & $<.001$ \\
\hline & ICU & 40 & 40 & .93 \\
\hline & $28 / 30$ & 34 & 29 & .046 \\
\hline & 60 & 38 & 26 & $<.001$ \\
\hline $\begin{array}{l}\mathrm{A}=\text { befor } \\
\mathrm{B}=1995 \\
\mathrm{C}=\text { after } \\
\text { Prospectiv } \\
\text { RCTs }=\mathrm{r} \\
\mathrm{NA}=\text { not }\end{array}$ & $\begin{array}{l}\text { spective observa } \\
\text { zed controlled tri } \\
\text { ble }\end{array}$ & tudies & & \\
\hline
\end{tabular}

either acute or chronic comorbidities. The cause of death in patients with ARDS is usually not lung pathology per se but mostly concurrent extrapulmonary organ dysfunction. Early mortality is often related to the causal factor of the lung injury, and late mortality is more associated with complications (eg, sepsis and multipleorgan system failure). ${ }^{11,12}$

From the first clinically relevant description of acute lung injury/ARDS in $1967^{13}$ to the early 1990s, the epidemiological data often yielded ambiguous and biased results due to the lack of generally accepted diagnostic criteria. The studies also included subjects with infantile respiratory distress syndrome, acute kidney injury, fluid overload, drug intoxication, disseminated intravascular coagulation, and burns. ${ }^{14}$ AECC criteria established in 1994 enhanced the diagnostic accuracy of the syndrome and facilitated research in the field. However, many articles published in this period reported mortality rates in a surprisingly wide range from 15 to $72 \% .^{6,15-25}$ Our findings confirmed the wide span of mortality rates during the time period (ie, from 13 to $80 \%$ in the specific group and from 11 to $87 \%$ in the mixed group). The third period (since 2001) is characterized by the implementation of lung-protective mechanical ventilation positively affecting the mortality of ARDS. ${ }^{9}$

The mentioned mortality differences could be explained by a type of the causal pathologic condition (eg, sepsis and hospital-acquired/ventilator-associated pneumonia are associated with higher mortality, whereas trauma-related injury or aspiration is associated with a lower mortality).26,27 The incidence of nosocomial infections varies among regions, ${ }^{28}$ with the type of pathogen (viruses vs bacteria, antibiotic-sensitive vs multidrug-resistant bacteria) also playing a significant role..$^{29,30}$ The other explanations are as follows: population-based features (eg, composition of genetic factors, age, and sex); environmental features (eg, level of economic development, prevailing type of industry, local environment, cultural habits); health-care system differences (eg, health-care policy and area coverage, type of health insurance, and financial resources); research-related factors (eg, variability in the process of diagnosis; time factors [length of the enrollment, years vs months]; number of centers and ICUs involved; number of ICU beds; and type of data collection, processing, and storage). More reasons for the mortality-related differences could be (1) the data acquisition in various regions during specific time periods (ie, the changes in etiological patterns during year [incidence of viral infections in late winter, incidence of multiple traumas during warm seasons]; (2) the incomplete application of favorable interventions for patients with ARDS in routine practice; (3) the absence of discrimination between the terms acute lung injury and ARDS in diagnostic process or using the inappropriate term "acute respiratory failure"; (4) changes in the frequency of specific forms of ARDS (eg, due to outbreaks of various infectious diseases [severe acute respiratory syndrome or influenza A H1N1 2009]); (5) gradual increases in mean age and the number of serious comorbidities of 

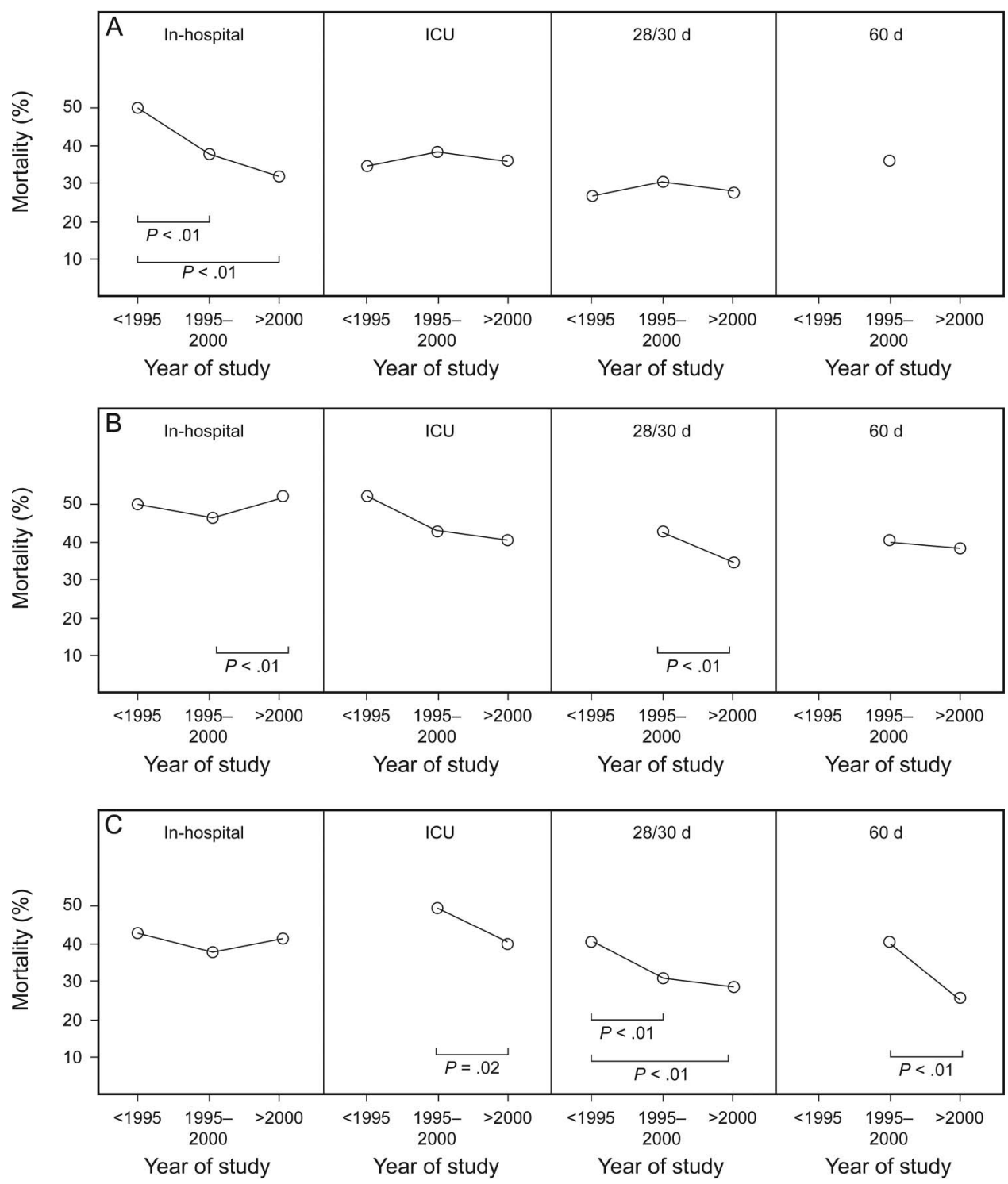

Fig. 3. Weighted averages of reported mortality rates in retrospective studies (A), prospective studies (B), and randomized controlled trials (C). Reported mortality types are in-hospital, ICU, 28/30-d, and 60-d mortality.

Table 5. Absolute Values of the Most Recent Mortality Rates, Since 2010

\begin{tabular}{|c|c|c|c|c|c|c|c|c|}
\hline \multirow{2}{*}{$\begin{array}{l}\text { Mortality } \\
\text { Type }\end{array}$} & \multicolumn{2}{|c|}{ Retrospective } & \multicolumn{2}{|c|}{ Prospective } & \multicolumn{2}{|c|}{ RCTs } & \multicolumn{2}{|c|}{ All } \\
\hline & $n$ & $\%$ & $n$ & $\%$ & $n$ & $\%$ & $n$ & $\%$ \\
\hline In-hospital & 345 & 50 & 759 & 47 & 1,060 & 41 & 2,164 & 45 \\
\hline ICU & 547 & 33 & 340 & 46 & 898 & 39 & 1,785 & 38 \\
\hline 28/30-d & 1,068 & 26 & 606 & 34 & 832 & 31 & 2,506 & 30 \\
\hline $60-d$ & 751 & 33 & 1,294 & 38 & 858 & 23 & 2,903 & 32 \\
\hline
\end{tabular}

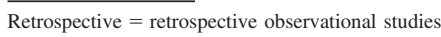

Prospective $=$ prospective observational studies

RCTs $=$ randomized controlled trials subjects; and (6) differences in the general hospital management of subjects after discharge from ICU, which might contribute up to $3-15 \%$ of the overall mortality. ${ }^{31,32}$ These mentioned factors might also limit our analysis and findings. Moreover, we observed that the types of mortality rates reported by studies often vary (eg, overall, 28-d, 30-d, 60-d, 90-d, in-hospital, ICU, 8-week), which significantly contributed to the inconsistency among clinical studies. The 28/30-d mortality and 60-d mortality are qualitatively different from ICU and in-hospital mortality. We suggest that due to the extended hospital stay of patients with ARDS, the ICU and especially in-hospital mortality are close to or may even be longer than $28 / 30 \mathrm{~d}$. The 60 -d 
mortality is described in a limited number of studies mostly reporting just one type of mortality. One can see in Figure 3 that in the corresponding time periods, in almost all cases, overall $28 / 30$-d mortality is lower than 60 -d mortality, as could be expected. The same applies also for ICU and in-hospital mortality. The particular study methodology could have a significant influence on reported mortality values and might be a source of bias.

Given the known problems of static mortality data, an analysis of the kinetics (trends) of mortality rates over time ${ }^{33-38}$ seems more valuable. In the period before 1994, several studies reported steady mortality rates $(51 \pm 19 \%)$ from 1967 to $1994 .{ }^{39}$ However, Milberg et al ${ }^{40}$ reported different results, describing a steady state of mortality from 1983 to 1987 and a subsequent decline until 1993. In another single-center study, the cohorts of subjects in specific years (1982, 1990, 1994, and 1998) presented a decrease in overall mortality, from $68 \%$ in 1982 to $29 \%$ in 1996, with a plateau in the mid-1990s. ${ }^{41}$ In 2008, Zambon and Vincent ${ }^{15}$ analyzed 72 studies, including a total number of 11,426 subjects, and described a tendency for mortality to decrease from 1994 to 2006 . They reported a wide range of mortality rates, from 15 to $72 \%$, with an overall pooled mortality of $43 \%$. They observed reductions in mortality in both interventional and non-interventional studies (trials with and without inclusion/exclusion criteria, respectively). The authors suggested that the improvement in survival may not have only been due to better respiratory management, but several other non-pulmonary factors could also have played a role (eg, better timing and rationalization of therapeutic interventions [antibiotic administration, fluid therapy, blood transfusions], glucose control, hygienic measures, improved infection source control and overall sepsis management). ${ }^{15}$ The second study challenged the decrease in mortality trends. It analyzed data from 89 studies conducted between 1984 and 2006, including 53 observational and 36 randomized trials with a total of 18,900 subjects. The reported overall pooled mortality rate was $44 \%$. The observational studies showed a decrease in mortality until 1993, but the overall mortality did not decrease from 1994 to 2006 . The mortality value was lower in RCTs (36\%) than it was in observational studies $(44 \%) .{ }^{16} \mathrm{We}$ subdivided the studies in greater detail according to time, study methodology, and reported type of mortality rate. In contrast to Phua et al, ${ }^{16}$ we found a significant decreasing tendency for in-hospital mortality in retrospective observational studies, ICU mortality in RCTs, and 28/30-d mortality in prospective observational studies and RCTs over all 3 followed time periods. Fur- thermore, Phua et al ${ }^{16}$ found a significant difference between observational and randomized trials (including prospective studies and both interventional and non-interventional arms of RCTs). We did not conclusively confirm their results, because only 5 of 9 of calculable subgroups (56\%) were significantly different. The reason for the difference might be that we analyzed a different number of studies (139 vs 89 , respectively) including more subjects $(25,996$ vs 18,900$)$. Moreover, we subdivided the studies according to mortality type and deliberately did not include interventional arms of RCTs due to possible influence of particular intervention on mortality results.

Moreover, we performed a comparison based on prospectivity versus retrospectivity. We found that prospective studies (prospective observational studies and noninterventional arms of RCTs considered together) report significantly higher mortality rates only in 4 of 10 calculable subgroups (40\%) compared with retrospective observational studies. Thus, we did not confirm the presumed disadvantage of retrospective data collection: the inability to enroll all eligible patients and missing opportunity to collect all required data.

In the regression model, we found that the number of centers negatively correlated with reported mortality. The reason might be that the studies including more centers enrolled fewer patients per center with more focused management considered as a modified Hawthorne effect within medical staff. The length of the time interval of subjects' enrollment has no significant influence on reported mortality. We suggest that the high variability of the length of studies prevents the demonstration of significance. The linear tendency of mortality to decrease over time might be considered as the most important finding.

Finally, to present the most recent mortality data, we additionally calculated the overall rates for in-hospital, ICU and 28/30-d and 60-d mortality $(45,38,30$, and 32\%, respectively) since 2010 . We suggest these values as possible benchmarks for further clinical trials reporting the particular types of mortality.

Due to our experience with adult patients only, we omitted results from pediatric studies, and age $<18$ y of the subjects was one of the exclusion criteria. However, we are aware of the fact that children of adolescent age are somatically almost identical to adults and could have been included. It might be considered as a limitation of this review. However, we were afraid of problems that might have been caused by including the data of school-age and younger children with different physiology and ARDS risk factors compared with adults. 


\section{Mortality of ARDS: A Systematic Review}

\section{Conclusions}

The reported mortality rates are highly heterogeneous and often biased, and their interpretation has many limitations. We advise against the unqualified adoption of reported mortality data from different studies performed in different time periods. Comparing these data with routine practice could be misleading, and using them in designing new clinical trials could diminish the value of the research results. We suggest carefully taking into account the study methodology (observational vs randomized and retrospective vs prospective, specific vs mixed ARDS population), with preference given to prospective modes of data collection and critical consideration of the age and length of the study. The mortality trends suggest a decreasing tendency in prospectively designed studies over time; however, mortality remains high.

\section{ACKNOWLEDGMENTS}

We thank Dr E David G McIntosh (Imperial College, London, United Kingdom) for the final correction of the manuscript.

\section{REFERENCES}

1. Bernard GR, Artigas A, Brigham KL, Carlet J, Falke K, Hudson L, et al. The American-European Consensus Conference on ARDS: definitions, mechanism, relevant outcomes, and clinical trial coordination. Am J Respir Crit Care Med 1994;149(3 Pt 1):818-824.

2. Artigas A, Bernard GR, Carlet J, Dreyfuss D, Gattinoni L, Hudson L, et al. The American-European Consensus Conference on ARDS, part 2: ventilatory, pharmacologic, supportive therapy, study design strategies, and issues related to recovery and remodeling. Acute respiratory distress syndrome. Am J Respir Crit Care Med 1998;157(4 Pt 1):1332-1347.

3. ARDS Definition Task Force, Ranieri VM, Rubenfeld GD, Thompson BT, Ferguson ND, Caldwell E, Fan E, Camporota L, Slutsky AS. Acute respiratory distress syndrome: the Berlin Definition. JAMA 2012;307(23):2526-2533.

4. Fanelli V, Vlachou A, Ghannadian S, Simonetti U, Slutsky AS, Zhang H. Acute respiratory distress syndrome: new definition, current and future therapeutic options. J Thorac Dis 2013;5(3):326-334.

5. Hamel MB, Phillips RS, Davis RB, Teno J, Connors AF, Desbiens $\mathrm{N}$, et al. Outcomes and cost-effectiveness of ventilator support and aggressive care for patients with acute respiratory failure due to pneumonia or acute respiratory distress syndrome. Am J Med 2000; 109(8):614-620.

6. Rubenfeld GD, Caldwell E, Peabody E, Weaver J, Martin DP, Neff $\mathrm{M}$, et al. Incidence and outcomes of acute lung injury. N Engl J Med 2005;353(16): 1685-1693

7. Tonelli AR, Zein J, Adams J, Ioannidis JP. Effects of interventions on survival in acute respiratory distress syndrome: an umbrella review of 159 published randomized trials and 29 meta-analyses. Intensive Care Med 2014;40(6):769-787.

8. Moher D, Liberati A, Tetzlaff J, Altman DG, PRISMA Group. Preferred reporting items for systematic reviews and meta-analyses: the PRISMA statement. PLoS Med 2009;6(7):e1000097.

9. Acute Respiratory Distress Syndrome Network. Ventilation with lower tidal volumes as compared with traditional tidal volumes for acute lung injury and the acute respiratory distress syndrome. N Engl J Med 2000;342(18):1301-1308.
10. R Core Team. R: A language and environment for statistical computing. Vienna, Austria: R Foundation for Statistical Computing; 2015. http://www.R-project.org/.

11. Villar J, Blanco J, Añón JM, Santos-Bouza A, Blanch L, Ambrós A, et al. The ALIEN study: incidence and outcome of acute respiratory distress syndrome in the era of lung protective ventilation. Intensive Care Med 2011;37(12):1932-1941.

12. Bernard GR. Acute respiratory distress syndrome: a historical perspective. Am J Respir Crit Care Med 2005;172(7):798-806.

13. Ashbaugh DG, Bigelow DB, Petty TL, Levine BE. Acute respiratory distress in adults. Lancet 1967;2(7511):319-323.

14. Murray JF, Staff of the Division of Lung Diseases, Heart, Lung and Blood Institute. Mechanisms of acute respiratory failure. Am Rev Respir Dis 1977;115(6):1071-1078.

15. Zambon M, Vincent JL. Mortality rates for patients with acute lung injury/ARDS have decreased over time. Chest 2008;133(5):11201127.

16. Phua J, Badia JR, Adhikari NK, Friedrich JO, Fowler RA, Singh JM, et al. Has mortality from acute respiratory distress syndrome decreased over time? Am J Respir Crit Care Med 2009;179(3):220-227.

17. Ferguson ND, Frutos-Vivar F, Esteban A, Anzueto A, Alía I, Brower RG, et al. Airway pressures, tidal volumes, and mortality in patients with acute respiratory distress syndrome. Crit Care Med 2005;33(1): 21-30.

18. Esteban A, Ferguson ND, Meade MO, Frutos-Vivar F, Apezteguia C, Brochard L, et al. Evolution of mechanical ventilation in response to clinical research. Am J Respir Crit Care Med 2008;177(2):170-177.

19. Rubenfeld GD. Epidemiology of acute lung injury. Crit Care Med 2003;31(4 Suppl):S276-S284.

20. Cunningham AJ. Acute respiratory distress syndrome-two decades later. Yale J Biol Med 1991;64(4):387-402.

21. Webster NR, Cohen AT, Nunn JF. Adult respiratory distress syndrome: how many cases in the UK? Anaesthesia 1988;43(11):923926.

22. Villar J, Slutsky AS. The incidence of the adult respiratory distress syndrome. Am Rev Respir Dis 1989;140(3):814-816.

23. Dushianthan A, Grocott MP, Postle AD, Cusack R. Acute respiratory distress syndrome and acute lung injury. Postgrad Med J 2011; 87(1031):612-622.

24. Valta P, Uusaro A, Nunes S, Ruokonen E, Takala J. Acute respiratory distress syndrome: frequency, clinical course, and costs of care. Crit Care Med 1999;27(11):2367-2374.

25. Linko R, Okkonen M, Pettila V, Perttilä J, Parviainen I, Ruokonen E, et al. Acute respiratory failure in intensive care units: FINNALI: a prospective cohort study. Intensive Care Med 2009;35(8):1352-1361.

26. Montgomery AB, Stager MA, Carrico CJ, Hudson LD. Causes of mortality in patients with the adult respiratory distress syndrome. Am Rev Respir Dis 1985;132(3):485-489.

27. Stapleton RD, Wang BM, Hudson LD, Rubenfeld GD, Caldwell ES, Steinberg KP. Causes and timing of death in patients with ARDS. Chest 2005;128(2):525-532.

28. Vincent JL, Sakr Y, Sprung CL, Ranieri VM, Reinhart K, Gerlach H, et al. Sepsis in European intensive care units: results of the SOAP study. Crit Care Med 2006;34(2):344-353.

29. Ruuskanen O, Lahti E, Jennings LC, Murdoch DR. Viral pneumonia. Lancet 2011;377(9773):1264-1275.

30. Kalanuria AA, Ziai W, Mirski M. Ventilator-associated pneumonia in the ICU. Crit Care 2014;18(2):208

31. Fialkow L, Vieira SR, Fernandes AK, Silva DR, Bozzetti MC, Acute Respiratory Distress Syndrome Research Group. Acute lung injury and acute respiratory distress syndrome at the intensive care unit of a general university hospital in Brazil: an epidemiological study using the American-European Consensus Criteria. Intensive Care Med 2002;28(11):1644-1648. 


\section{Mortality of ARDS: A Systematic Review}

32. Esteban A, Alia I, Gordo F, de Pablo R, Suarez J, González G, Blanco J. Prospective randomized trial comparing pressure-controlled ventilation and volume controlled ventilation in ARDS. Chest 2000; 117(6): 1690-1696.

33. Tang SS, Redmond K, Griffiths M, Ladas G, Goldstraw P, Dusmet M. The mortality from acute respiratory distress syndrome after pulmonary resection is reducing: a 10-year single institutional experience. Eur J Cardiothorac Surg 2008;34(4):898-902.

34. Martin M, Salim A, Murray J, Demetriades D, Belzberg H, Rhee P. The decreasing incidence and mortality of acute respiratory distress syndrome after injury: a 5-year observational study. J Trauma 2005; 59(5):1107-1113.

35. Rocco TR Jr, Reinert SE, Cioffi W, Harrington D, Buczko G, Simms HH. A 9-year, single-institution, retrospective review of death rate and prognostic factors in adult respiratory distress syndrome. Ann Surg 2001;233(3):414-422.

36. Abel SJ, Finney SJ, Brett SJ, Keogh BF, Morgan CJ, Evans TW. Reduced mortality in association with the acute respiratory distress syndrome (ARDS). Thorax 1998;53(4):292-294.
37. Sigurdsson MI, Sigvaldason K, Gunnarsson TS, Moller A, Sigurdsson $\mathrm{GH}$. Acute respiratory distress syndrome: nationwide changes in incidence, treatment and mortality over 23 years. Acta Anaesthesiol Scand 2013;57(1):37-45.

38. Pola MD, Navarrete-Navarro P, Rivera R, Fernández-Mondejar E, Fernández-Mondejar E, Hurtado B, Vázquez-Mata G. Acute respiratory distress syndrome: resource use and outcomes in 1985 and 1995, trends in mortality and comorbidities. J Crit Care 2000;15(3): 91-96.

39. Krafft P, Fridrich P, Pernerstorfer T, Fitzgerald RD, Koc D, Schneider B, et al. The acute respiratory distress syndrome: definitions, severity and clinical outcome: an analysis of 101 clinical investigations. Intensive Care Med 1996;22(6):519-529.

40. Milberg JA, Davis DR, Steinberg KP, Hudson LD. Improved survival of patients with acute respiratory distress syndrome (ARDS): 1983-1993. JAMA 1995;273(4):306-309.

41. Zilberberg MD, Epstein SK. Acute lung injury in the medical ICU: comorbid conditions, age, etiology, and hospital outcome. Am J Respir Crit Care Med 1998;157(4 Pt 1):1159-1164. 